\title{
CLINICAL PATTERN OF FIXED DRUG ERUPTIONS IN A TERTIARY CARE HOSPITAL OF SOUTHERN ASSAM: A PROSPECTIVE STUDY
}

Mahimanjan Saha1, Bhaskar Gupta², Debajit Das³, Joydeep Roy4, Arup Paul5, Ashis Dey6, Vaswatee Madhab7

\section{HOW TO CITE THIS ARTICLE:}

Mahimanjan Saha, Bhaskar Gupta, Debajit Das, Joydeep Roy, Arup Paul, Ashis Dey, Vaswatee Madhab. "Clinical Pattern of Fixed Drug Eruptions in a Tertiary Care Hospital of Southern Assam: A Prospective Study". Journal of Evolution of Medical and Dental Sciences 2015; Vol. 4, Issue 69, August 27; Page: 11936-11943,

DOI: $10.14260 /$ jemds/2015/1723

ABSTRACT: BACKGROUND: Fixed drug eruption (FDE) is a type of very common cutaneous adverse drug reaction seen in day to day practices. It is very embarrassing to patients due to repeated recurrences and may cause cosmetic problem when present on exposed surface. AIMS: To study the clinical pattern of FDE and identify drugs commonly causing it among patients attending in a tertiary care hospital of southern Assam. MATERIALS AND METHODS: An observational, prospective study was conducted in 1 year period from $1^{\text {st }}$ September 2013 to 31st August 2014 in a tertiary care hospital of southern Assam. 86 patients of suspected fixed eruption due to drugs included in the study. Proper history with clinical evaluation were done and recorded as per structured pro forma by Central Drugs Standard Control Organization (CDSCO). Along with identification of causative drugs, morphological and distribution pattern of FDE studied extensively. The significance of preferential site involvement due to particular drug was statistically evaluated by Fischer's exact test. RESULTS: Cotrimoxazole (33.7\%) was the major etiological agent in causing FDE in the southern part of Assam, followed by NSAIDS (20.9\%), Tetracyclines (12.8\%), Ornidazole (11.6\%), Ciprofloxacin (9.3\%) and Metronidazole (8.1\%). On oral provocation, $80 \%$ patients showed positive response to offending drugs. Few patients (29\%) had bullous FDE, of which Cotrimoxazole was the offending agent, followed by Doxycycline. In males oro-genital mucosa was commonly affected; whereas in females the lesions were distributed among the trunk and extremities. Cotrimoxazole induced involvement of mucosa and NSAIDS induced involvement of trunk and extremities both are statistically significant. CONCLUSIONS: The clinical pattern and drugs causing FDE were almost similar to those observed in previous studies except for some minor variations, which may be due to the differences in regional variations and changing trends of pharmacy.

KEYWORDS: Fixed drug eruption, Cutaneous adverse drug reaction, Cotrimoxazole.

INTRODUCTION: "Anything you can think of, anything you can see, and some things you don't even think of can be due to a drug." E. D. Shelley \& W. B. Shelley.

Adverse drug reaction may be defined as 'an undesirable clinical manifestation resulting from administration of particular drug; this includes reaction due to overdose, predictable side effects and unanticipated adverse manifestations'.[1] All cutaneous eruption are distinct disease entities and different groups of drugs are associated with different types of eruption. Brocq in 1984, coined the term 'fixed eruption' to describe a specialized pattern of skin eruption due to antipyrine.[2] Fixed drug eruptions (FDE) is characterized by development of one or more annular or oval, sharply demarcated erythematous plaques or blisters as a result of systemic exposure to a drug, which usually leaves a residual hyperpigmentation.[1],[3]They classically recurs at the same site, with each exposure of same medication with involvement of new areas in repeated exposure.[1],[3] 
The presence of numerous lesions as a consequence of repeated exposure is referred to as generalized FDE, and it may be difficult to distinguish from erythema multiforme or Stevens-Johnson syndrome(SJS) especially when oral mucosa is involved.[4]Non-pigmenting variant of FDE is uncommon and mainly seen with sympathomimetics and Non-steroidal anti-inflammatory drugs(NSAID).[1],[4]

Numerous drugs can cause FDE and cross-sensitivity between drugs of same group is also reported. [5] Causative drugs and pattern of distribution varies from country to country, over time to time.[3] This is because of difference in the health seeking behaviour of the individuals of different countries, further day by day new drugs are coming and some older drugs are withdrawn from pharmaceutical market.

In this background, it was thought appropriate to study clinical pattern of FDE attending our institute.

MATERIALS AND METHODS: An observational, prospective study was conducted in 1 year period from $1^{\text {st }}$ September 2013 to 31st August 2014 in the Dermatology department of Silchar medical college, Silchar. The study was approved by the Institutional Ethics Committee.

All patients of FDE attending this department (Either self-presenting or referred by other departments of this institution), of both gender, under all age group were included. Suspected fixed eruptions due to non-drug causes (Food particles e.g., Cashew, liquorice), patients with incomplete drug history and other cutaneous eruption caused by drugs (Stevens-Johnson syndrome, Lyell's syndrome) were excluded from the study.

A detailed history regarding drug intake, mode of drug intake, type of illness for which the drug was taken, total number of dose taken, whether had been prescribed by a Registered Medical practitioner or taken over the counter (OTC), were evaluated. A through clinical evaluation was carried out and the morphological patterns of lesions-bullous/nonbullous and pattern of distribution- skin/mucosa were noted. For diagnosis of etiological agents, along with drug history, temporal correlation with drug intake and approximate incubation period noted. Effect of withdrawal of drugs (Dechallange) causing clinical improvement observed in all patients whereas reactivation of lesions on Rechallange in the form of oral provocation test was done wherever feasible.

All the data were recorded as per ADR form obtained from Central Drugs Standard Control Organization (CDSCO) website, www.cdsco.nic.in. The significance of preferential site involvement due to particular drug was statistically evaluated by Fischer's exact test. $\mathrm{P}$ value $<0.05$ was considered statistically significant.

RESULTS AND DISCUSSION: Over the period of 1 year, total 92 cases of fixed eruption due to drugs diagnosed in patients attending dermatology department of our institute. 6 cases were excluded from further analysis: 2 refused to give consent and 4 patients failed to recall the correct name of medication consumed. Among the remaining 86 cases, there were 45 males (52.3\%) and 41 females $(47.3 \%)$. The age of patients ranged from 1 year to 75 years (mean=31.6). Both males and females were predominant among the age group between 30 to 39 years. The distribution of cases in relation to age and gender are provided in Table-1 and Graph-1. 


\section{ORIGINAL ARTICLE}

\begin{tabular}{|c|c|c|c|}
\hline Age in Years & Males & Females & Total \\
\hline$<9$ & 4 & 3 & 7 \\
\hline $10-19$ & 7 & 5 & 12 \\
\hline $20-29$ & 8 & 9 & 17 \\
\hline $30-39$ & 11 & 12 & 23 \\
\hline $40-49$ & 10 & 8 & 18 \\
\hline 50 \& Above & 5 & 4 & 9 \\
\hline Total & 45 & 41 & 86 \\
\hline
\end{tabular}

Table 1: Distribution of cases in relation to age and gender

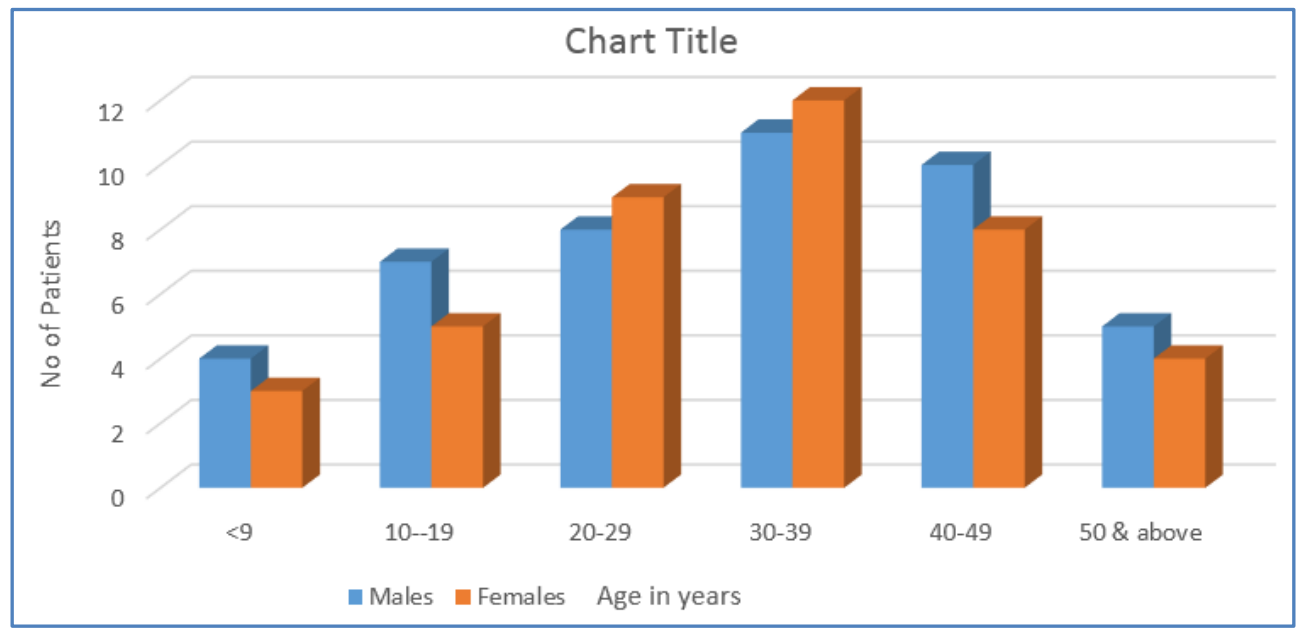

Graph 1: Distribution of cases in relation to age and gender

A slight male preponderance was observed (M: $F=1.09: 1)$ which was previously reported by VK Sharma et al.[6] Persons around 11-40 years are commonly affected which are in concordance with Mehta et al 1971.[7]

Causative Drugs of FDE: The causative drugs for FDE are shown in Table -2 .

\begin{tabular}{|c|c|c|}
\hline Drugs & No. of Patients & Percentage (\%) \\
\hline COTRIMOXAZOLE & 29 & 33.7 \\
\hline NSAIDS & 18 & 20.9 \\
\hline TETRACYCLINES & 11 & 12.8 \\
\hline ORNIDAZOLE & 10 & 11.6 \\
\hline CIPROFLOXACIN & 8 & 9.3 \\
\hline METRONIDAZOLE & 7 & 8.1 \\
\hline CEPHALOSPORINS & 2 & 2.3 \\
\hline AMOXYCILLIN & 1 & 1.2 \\
\hline TOTAL & 86 & 100 \\
\hline
\end{tabular}




\section{ORIGINAL ARTICLE}

NSAIDS- Acetaminophen, Ibuprofen, Nimesulide

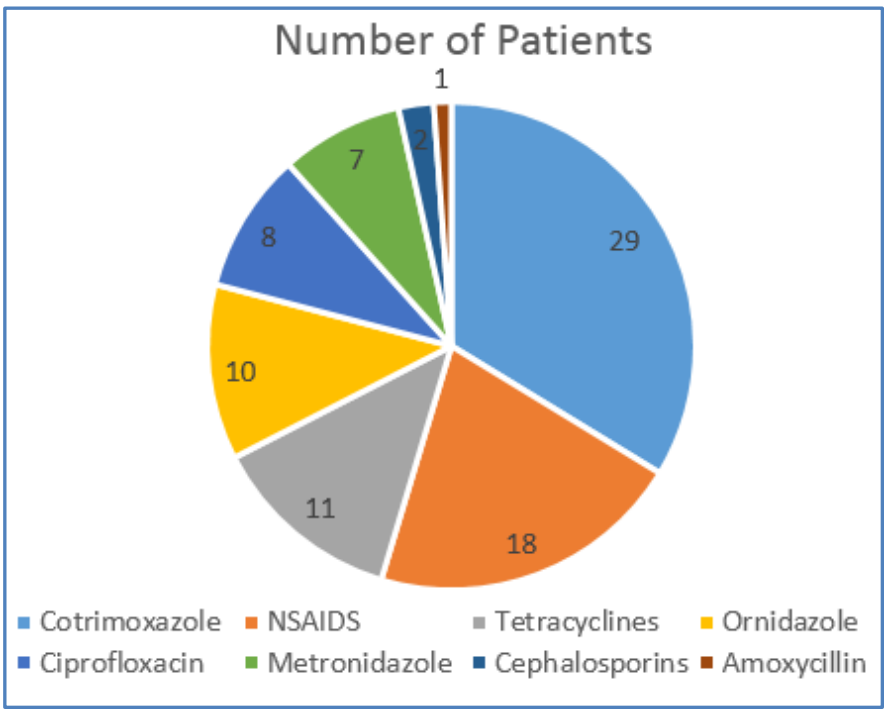

Graph 2: Drugs causing fixed drug eruptions

The drugs were taken commonly for fever, headache, joint pain, dysentery, tooth-ache, upper respiratory tract infection. The incubation period varied between 30 minutes to 10 days. 18 patients $(20.9 \%)$ had taken the drug over the counter, without a proper prescription from a Registered Medical Practitioner.

\begin{tabular}{|c|c|c|c|}
\hline Author & Year & Commonest Drug & Percentage (\%) \\
\hline Thankappan TP et al[8] & 1991 & Cotrimoxazole & 36.3 \\
\hline Sharma VK et al[6] & 1996 & Cotrimoxazole & 32.8 \\
\hline Patel RM \& Marfatia YS[9] & 2008 & Cotrimoxazole & 29.5 \\
\hline Present study & $2013-2014$ & Cotrimoxazole & 33.7 \\
\hline \multicolumn{2}{|c|}{ Table 3: Most common drug causing FDE } \\
\hline
\end{tabular}

Most common drug causing FDE is Cotrimoxazole which is as per above studies. However Tetracyclines were reported to be commonest as per Parischa JS.[10] NSAIDS like phenazone (Antipyrine), Metamizole and barbiturates as revealed by some older studies as most frequent agents, not widely used now a days.[3],[11]

\begin{tabular}{|c|c|c|}
\hline Total No. of Cases & Positive (\%) & Negative (\%) \\
\hline 50 & $40(80 \%)$ & $10(20 \%)$ \\
\hline \multicolumn{3}{|c|}{ Table 4: Results of oral provocation test } \\
\hline
\end{tabular}

Rechallange (Oral provocation) test was conducted to identify the offending drugs. After taking proper consent, oral provocation done on 50 patients of which 40 patients (80\%) became positive as evidenced by erythema/or itching over preexisting lesion(reactivation).Test result corroborates with previous studies by Alanko et al (1989)[12] $-79.69 \%$ and Das J and Mandal AC

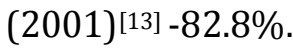




\section{ORIGINAL ARTICLE}

Morphology of Lesions: 20 patients had more than 5 lesions and Cotrimoxazole was the offending agent in 10.

25 patients (29\%) had bullous FDE, Cotrimoxazole being most common offending agents followed by Doxycycline. Size of the lesions ranged between $1-10 \mathrm{~cm}$. Lesions were mainly of a classical type presenting with sharply demarcated erythematous macules and occasional bulla formation.

Most of the solitary lesions on the glans penis were exclusively bullous of which some presented with erosion.

\section{Pattern of Distribution:}

\begin{tabular}{|c|c|c|c|c|c|}
\hline Drugs & Mucosa & Face & $\begin{array}{c}\text { Trunk \& } \\
\text { Extremities }\end{array}$ & $\begin{array}{c}\text { Acral } \\
\text { Lesions }\end{array}$ & Total \\
\hline Cotrimoxazole & $21^{*}$ & 2 & 6 & - & 29 \\
\hline Nsaids & 5 & - & $13^{*}$ & - & 18 \\
\hline Tetracyclines & 6 & - & 5 & - & 11 \\
\hline Ornidazole & 2 & 3 & 5 & & 10 \\
\hline Ciprofloxacin & 1 & 2 & 5 & - & 8 \\
\hline Metronidazole & 2 & - & 5 & - & 7 \\
\hline Cephalosporins & - & 1 & 1 & - & 2 \\
\hline Amoxycillin & - & - & - & 1 & 1 \\
\hline T0TAL & $\mathbf{3 7}$ & $\mathbf{8}$ & $\mathbf{4 0}$ & $\mathbf{1}$ & $\mathbf{8 6}$ \\
\hline \multicolumn{7}{|c|}{ Table 5: Localization of lesion } &
\end{tabular}

*Statistically very significant $\mathrm{P}$ value $=0.0043$.

Our study revealed, most frequently involved site was the oro-genital mucosa among males; trunk and extremities among females. Cotrimoxazole induced FDE were mainly located on the orogenital mucosa which is as per Kanwar et al[14] followed by trunk and extremities (Thankappan et al).[8] NSAIDS induced FDE mainly located in trunk and extremities which is as per VK Sharma et al 1998[15]. As per previous study,[6],[8] Tetracyclines was significantly associated with FDE in male genitalia. Amoxycillin induced FDE were confined to the palms and soles. Other drugs like ornidazole, ciprofloxacin and metronidazole had induced FDE more on the extremities.

CONCLUSION: From this study it was revealed, that Cotrimoxazole (33.7\%) was the major etiological agent in causing FDE in the southern part of Assam, followed by NSAIDS (20.9\%), Tetracyclines (12.8\%), Ornidazole (11.6\%), Ciprofloxacin (9.3\%) and Metronidazole (8.1\%).Few patients (29\%) had bullous FDE, of which Cotrimoxazole was the offending agent, followed by Doxycycline. In males oro-genital mucosa was commonly affected; whereas in females the lesions were distributed among the trunk and extremities. Cotrimoxazole induced involvement of mucosa and NSAIDS induced involvement of trunk and extremities both are statistically significant. Results of our study are in concordance to previous studies conducted both in India and abroad, except for minor variations which may be attributed by regional variations and changing trends in pharmacy. 


\section{ORIGINAL ARTICLE}

Treatment of FDE mainly symptomatic, consist of topical corticosteroids and antihistaminics. Repeated FDE over male genitalia causes great embarrassment to patients and hyperpigmentation after resolving FDE is also causes cosmetic concern when lesion present on exposed surface. Recurrence on repeated exposure is common feature and on each recurrences severity increases, so that offending drug should be identified early for betterment of the patients.

Patients should be properly educated about the nature of the disease and could be advised to carry a card containing the details of the drug to which they developed FDE or to wear a bracelet (eg: medic Alert) detailing the nature of the reaction and the drugs responsible for it to avoid unnecessary causality in the future.

Fig. 1: Cotrimoxazole induced FDE. On oral provocation test showing reactivation of lesion as evidenced by erythema at margins of preexisting lesion.

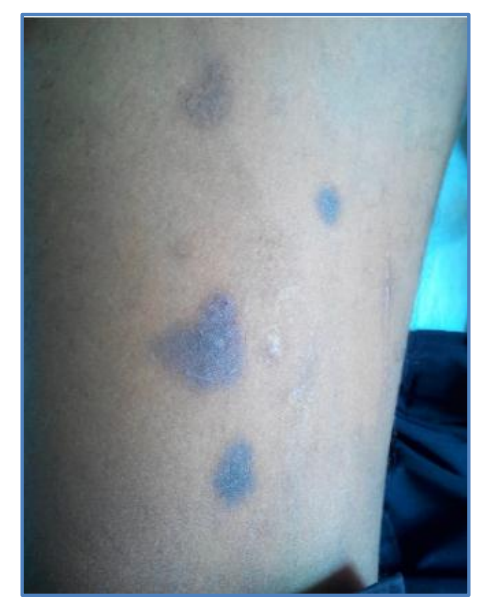

Fig. 1: Before Rechallange

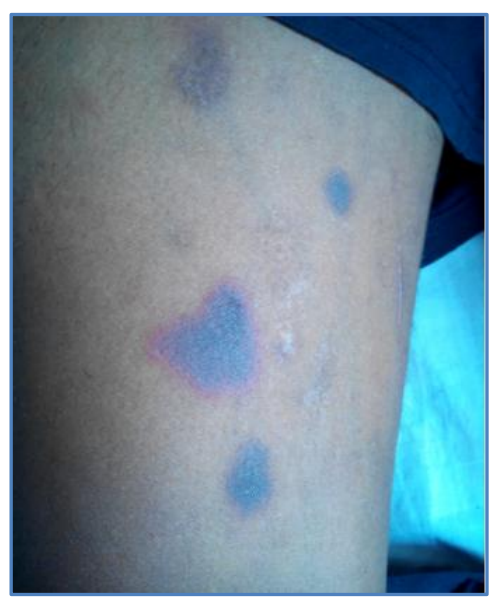

Fig. 1: After Rechallange

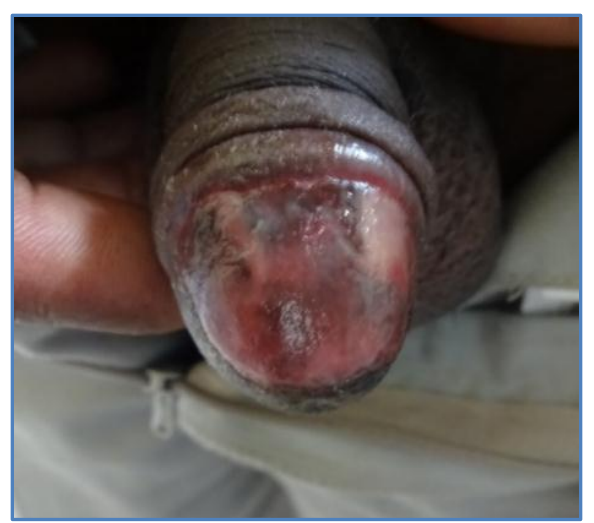

Fig. 2: Doxycycline induced FDE over glans penis

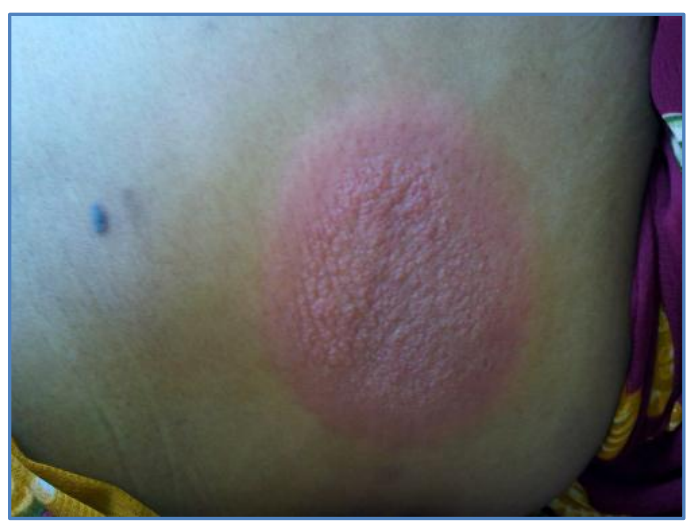

Fig. 3: Ornidazole induced FDE in acute stage 


\section{ORIGINAL ARTICLE}

\section{REFERENCES:}

1. Breathnach SM. Drug reactions. In: Burns T, Breathnach S, Cox N, Griffiths C, editors. Rook's Textbook of Dermatology. 8th $^{\text {th }}$ ed. Oxford: Wiley-Blackwell; 2010. p. 75.3-75.178.

2. Sovin JA. Current causes of fixed drug eruption: Br J Dermatol 1970; 80: 546-549.

3. Kauppinen K, Stubb S. Fixed eruptions: causative drugs and challenge tests. Br J Dermatol 1985; 112: 575-8.

4. Revuz J, Valeyrie-Allanore L. Drug Reactions. In: Bolognia JL, Jorrizo JL, Schaffer JV,editors.Dermatology,3rd ed. Philadelphia: Elsevier saunders;2012.p.335-56

5. Chan HL, Tan KC. Fixed drug eruption to three anticonvulsant drugs: an unusual case of polysensitivity. J Am Acad Dermatol 1997; 36: 259.

6. Sharma VK, Dhar S, Gill AN. Drug related involvement of specific sites in fixed eruptions: a statistical evaluation. J Dermatol 1996; 23: 530-4

7. Mehta TK, Marquis L, Shelty JN.A Study of seventy cases of drug eruptions. Indian journal of Dermatology, Venereology and Leprology.1971; 37:1-5.

8. Thankappan TP, Zachariah J. Drug specific clinical pattern in fixed drug eruptions. Int. J. Dermatol.1991; 30:867-70.

9. Patel RM, Marfatia YS. Clinical study of cutaneous drug eruptions in 200 patients. Indian J Dermatol Venereol Leprol 2008; 74:430.

10. Parischa JS. Drugs causing fixed drug eruptions.Br J Dermatol. 1979; 100; 183-185.

11. Kauppinen K. Cutaneous reactions to drugs. Acta Derm Venereol 1972; 52: 1-89

12. Alanko K, Stubb S, Kauppinen K. Cutaneous drug reactions: clinical types and causative agents. A five-year survey of in patients (1981-1985) Acta Derm Venereol. 1989; 69: 223-6.

13. Das J, Mandal A C. A study of drug eruptions by provocative tests. Indian J Dermatol Venereol Leprol 2001; 67: 238-9.

14. Kanwar AJ, Bharija SC, Singh M, and Belhaj MS. Ninety eight fixed drug eruptions with provocation tests. Dermatologica 1988; 177: 274-9.

15. Sharma V. K., G. Sethuraman, B. Kumar. Cutaneous Adverse drug reaction patterns to Antimicrobial drugs in North India. J. Assoc Physicians India 1998; 46:1012-15. 


\section{ORIGINAL ARTICLE}

\section{AUTHORS:}

1. Mahimanjan Saha

2. Bhaskar Gupta

3. Debajit Das

4. Joydeep Roy

5. Arup Paul

6. Ashis Dey

7. Vaswatee Madhab

\section{PARTICULARS OF CONTRIBUTORS:}

1. Post Graduate Resident, Department of Dermatology, Silchar Medical College.

2. Professor \& Head, Department of Dermatology, Silchar Medical College.

3. Associate Professor, Department of Dermatology, Silchar Medical College.

4. Registrar, Department of Dermatology, Silchar Medical College.

FINANCIAL OR OTHER COMPETING INTERESTS: None
5. Resident Physician, Department of Dermatology, Silchar Medical College

6. Assistant Professor, Department of Dermatology, Silchar Medical College

7. Post Graduate Resident, Department of Dermatology, Silchar Medical College.

\section{NAME ADDRESS EMAIL ID OF THE CORRESPONDING AUTHOR:}

Dr. Mahimanjan Saha, Post Graduate Resident, Department of Dermatology, Silchar Medical College, Silchar, Assam-788014, India.

E-mail:mahimanjan@gmail.com

Date of Submission: 12/08/2015. Date of Peer Review: 13/08/2015. Date of Acceptance: 24/08/2015. Date of Publishing: 25/08/2015. 